\title{
The effects of probiotic supplementation on emotional memory and pain response
}

The human intestinal microbiota is the ecological community of micro-organisms that share our gastrointestinal tract ${ }^{(1)}$. Increasing evidence suggests a mediating relationship exists between gut microbiota activity and the brain ${ }^{(2)}$. Recent research has shown that probiotic administration is capable of affecting brain activity in regions controlling central processing of emotion and sensation ${ }^{(3)}$. Developing on these findings, the aim of the present study was to assess the effect of probiotic administration on emotional memory (EM), anxiety and acute peripheral pain response (PR).

A randomised double-blind controlled trial, involving sixty participants aged 18-40 years, was conducted. Participants completed a six-week intervention, consuming either probiotic (Lactobacillus acidophilus CUL60 and CUL21, Bifidobacterium lactis CUL34, Bifidobacterium bifidum CUL20) or placebo (maltodextrin) capsules. Participants attended pre- and post-intervention sessions. Anxiety was assessed using the State-Trait Anxiety Inventory questionnaire at three time-points during each session (baseline, post-EM task, post-PR task), and again at weeks two and four during intervention. EM was assessed once in each session using a Remember, Know, Guess task; involving the presentation and recall of neutral and negative emotionally arousing images. A cold pressor test was used to assess PR; pain threshold, removal of hand and pain tolerance times were measured. A visual analogue scale was used to assess subjective pain. Finally, immune function was measured via salivary IgA, collected at three time-points in each session (baseline, post-EM task, post-PR task).

When comparing treatment groups in terms of EM, the probiotic group recalled significantly more negative images compared to placebo $(\mathrm{P}=0.022$, Figure 1.a). This effect was more pronounced in female participants $(\mathrm{P}=0.009$, Figure $1 . \mathrm{b})$. For male participants only, pain threshold was significantly reduced at the second assessment under the placebo condition $(-3.687$ seconds, $\mathrm{P}=0.027$, Figure 1.c) but not following probiotic administration, potentially indicating a protective effect of probiotic treatment on pain threshold. No further significant results were observed.

\section{GRAPH 1.a. REMEMBER RESPONSES PRE AND POST TREATMENT.}

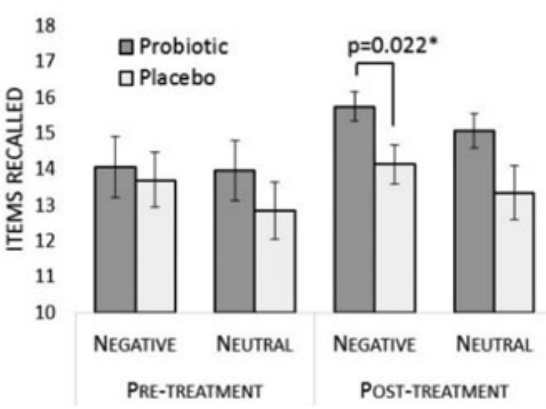

\section{GRAPH 1.b. REMEMBER RESPONSES PRE AND POST TREATMENTIN FEMALES.}

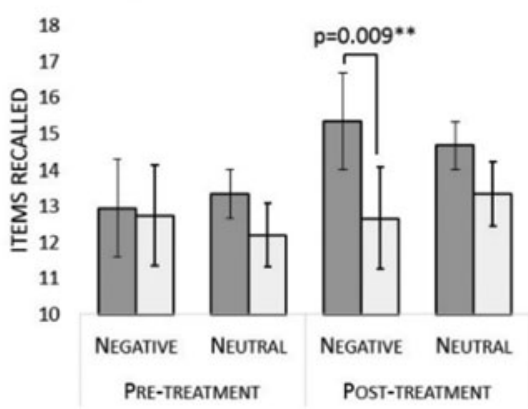

GRAPH 1.C PAIN THRESHOLD PRE AND POST TREAMENT IN MALES.

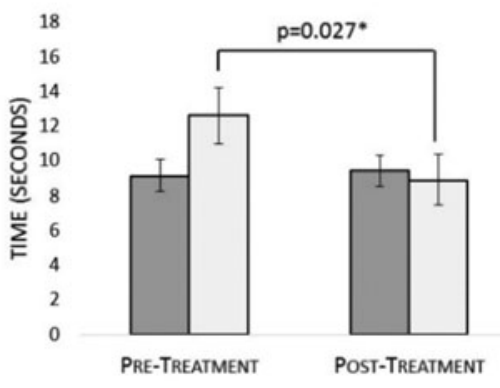

In conclusion, probiotic administration may have a moderating effect on EM processing. Specifically, augmented recall of highly negative valiant stimuli in the probiotic treatment group may indicate altered activity of brain regions that control central processing of emotion. Furthermore, probiotic treatment may have some potential to stabilise pain threshold in male participants. These initial data warrant further investigation to substantiate these findings.

This research was supported by Cultech LTD.

1. Cryan JF, Dinan TG (2012) Nat Rev Neurosci 13, 701-712

2. Forsythe P, Sudo N, Dinan T, et al. (2010) Brain Behav Immun 24, 9-16.

3. Tillisch K, Labus J, Kilpatrick L, et al. (2013) Gastroenterology 144, 1394-1401. 